\title{
Diagnosis of Kartagener Syndrome: a Rare Case
}

\author{
${ }^{1}$ Arismawati, ${ }^{2}$ Muhammad Hidayat Surya Atmaja ${ }^{*}$ \\ ${ }^{1}$ Resident and ${ }^{2}$ Staf of Radiology Department, Faculty of Medicine Dr. Soetomo General Academic Hospital, \\ Universitas Airlangga, Surabaya, Indonesia \\ *Corresponding author : hidayatsuryaatmaja@gmail.com
}

\begin{abstract}
Kartagener syndrome is a rare case. We report a 6-year-old pediatric patient with complaints of cough and shortness of breath. The patient had history of patent ductus arterious (PDA) and situs inversus. The patient underwent a chest x-ray examination which revealed situs inversus and bronchopneumonia. Waters X-ray revealed maxillary sinusitis. The patient also underwent a computed tomography (CT) scan, which revealed right lung bronchiectasis and situs inversus. Based on the patient's history and radiological examination results, the patient was diagnosed with Kartagener syndrome.
\end{abstract}

Keywords: Kartagener syndrome, situs inversus, bronchiectasis, sinusitis

\section{Introduction}

Primary ciliary dyskinesia (PCD) is a genetically heterogenous, autosomal recessive disease which are characterized by ciliary disfunction and impaired mucociliary clearance, resulting in various clinical manifestations. Based on situs inversus and bronchiectasis prevalence, its estimated to occur at 1/16,000 live births (Leigh et al, 2009).

Kartagener syndrome is a subset of PCD. It is a triad which consists of paranasal sinusitis, situs inversus, and bronchiectasis. It is first recognized in 1933 by Manes Kartagener. Its incidence is estimated to be $1 / 30,000$ live births and accounts for $1 / 6$ of situs inversus cases and $1 / 10$ of bronchiectasis cases consisting of situs inversus with bronchiectasis and paranasal sinusitis (Sarangi et al., 2016).

\section{Case Report}

A 6-year-old girl came to the hospital with complaints of cough with sputum, shortness of breath, and occasionally accompanied by fever. The patient had history of congenital heart defects (patent ductus arteriosus) and situs inversus. The laboratory test results showed an increase in leukocytes $(16,640 / \mathrm{mcl})$. Creactive protein (CRP) $0.6 \mathrm{mg} / \mathrm{dl}$ was within normal limit, Mantoux test revealed negative result, and GeneXpert test revealed no presence of Mycobacterium tuberculosis.

Radiological examinations were performed. Chest $\mathrm{x}$-ray revealed situs inversus and bronchopneumonia, while Water's view x-ray revealed left maxillary sinusitis (Figure 1). Chest computed tomography (CT) scan with contrast was also performed, where it revealed bronchiectasis of the right lung and situs inversus. Pneumonia and PDA were also found, which supported the diagnosis of Kartagener syndrome (Figure 2 and $3)$. 


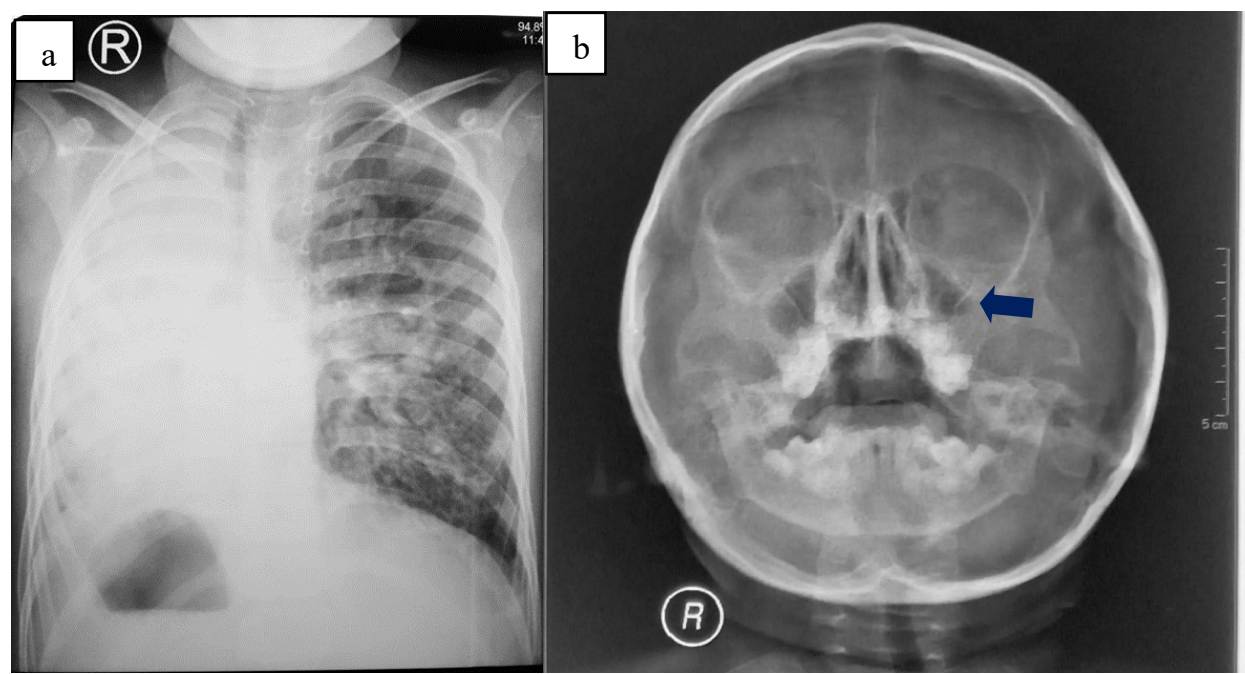

Figure 1. (a) Chest $\mathrm{x}$-ray revealed an impression that the heart is on the right side with consolidation in the right lung; (b) Water's view xray shows left maxillary sinusitis (blue arrow)
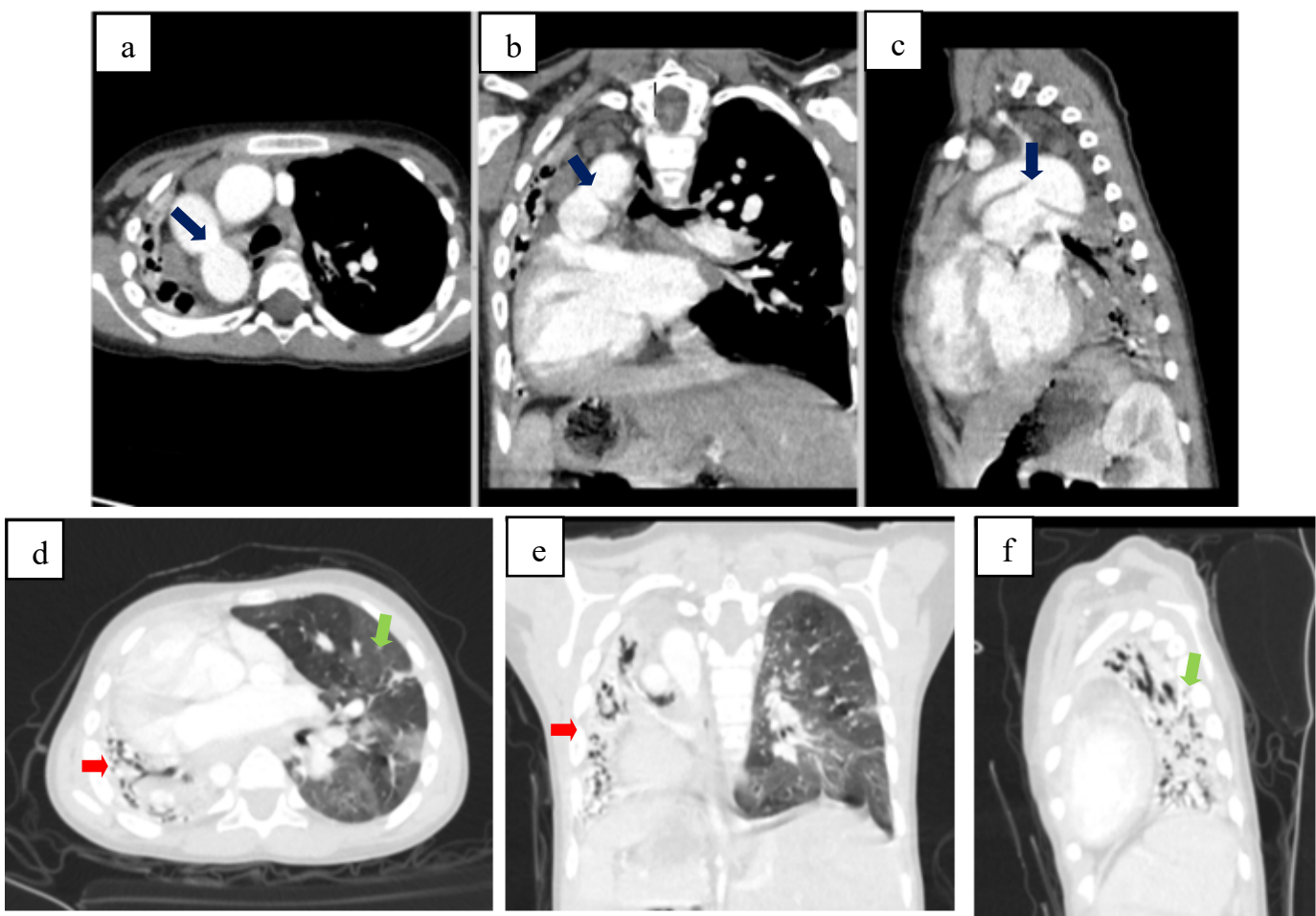

Figure 2. Chest CT Scan, axial (a), coronal (b), and sagittal view (c) on vein phase, showing PDA (blue arrow); Chest CT Scan, axial (d), coronal (e), and sagittal view (f) on lung window, showing bronchiectasis finding in the right lung (red arrow), alongside ground glass opacity in bilateral hemithorax (green arrow) 


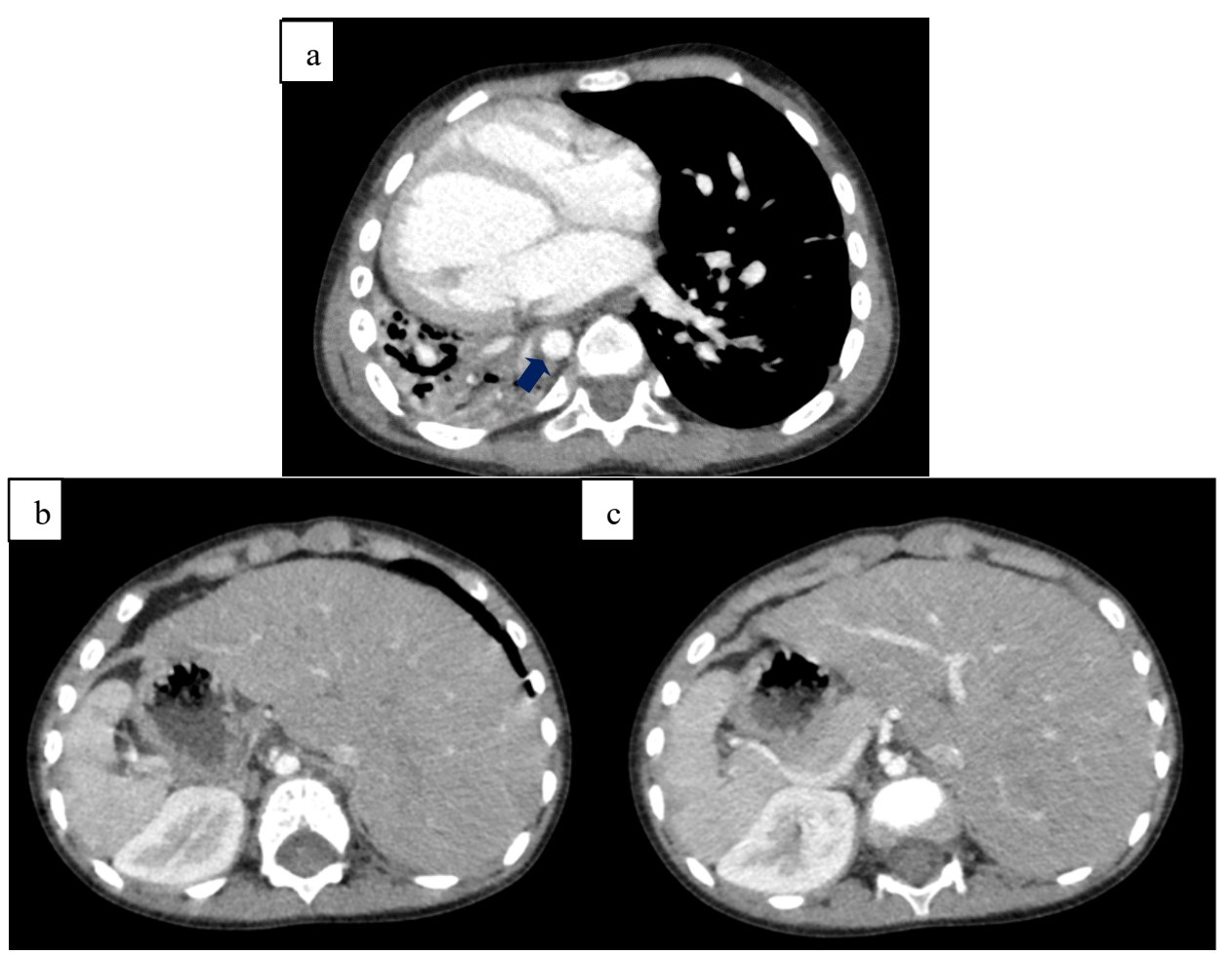

Figure 3. (a) Axial section of chest CT Scan with contrast on mediastinal window, showing descending aorta (blue arrow) on the right side with dextrocardia (situs inversus thoracalis); (b and c) Axial section of CT Scan with contrast on mediastinal window, showing transposition of abdominal visceral. Liver on the left side, spleen, stomach, and aorta on the right side (situs inversus abdominalis)

\section{Discussion}

Primary ciliary dyskenia's gold standard diagnostic test, including in Kartagener syndrome, is respiratory cilia electron microscopic ultrastructural analysis which is collected bronchial brush biopsy or nasal scrape. Several gene mutations were also identified, which are important for functional proteins in cilia. These genes are DNAI1, DNAH5, RSPH9, RSPH4A, KTU, DNAI2, DNAH11, and TXNDC3) (Babar et al., 2013; Leigh et al., 2009).

Patient's history and imaging investigation are important to facilitate early diagnosis if no gold standard test is important. A case report by Babar et al reported a 37 year old patient with bronchiectasis who had history of recurrent sinusitis and situs inversus. The findings were confirmed with radiological examinations and the patient was diagnosed with Kartagener syndrome (Babar et al., 2013). Another case report from Liu et al reported a 22 year old patient with a chief complaint of recurrent cough with yellow for 2 years and a history of paranasal sinusitis. Radiological examinations with CT scan revealed situs inversus, bronchiectasis, and sinusitis, which met Kartagener syndrome's triad (Liu et al., 2020). In the current case, the patient was admitted with complaints of cough with sputum, shortness of breath, and occasionally accompanied by fever. The patient had history of congenital heart defects (patent ductus arteriosus) and situs inversus. Radiological examinations with plain x-ray and $\mathrm{CT}$ scan with contrast revealed situs inversus, maxillary sinusitis, and bronchiectasis, which fulfilled the criteria for Kartagener syndrome diagnosis. 
There are several differential diagnoses for Kartagener syndrome, which include interstitial lung diseases, malignancies, and other conditions associated with bronchiectasis (Skeik and Jabr, 2011). The differential diagnosis is outlined in Table 1.

Table 1. Kartagener syndrome's differential diagnosis

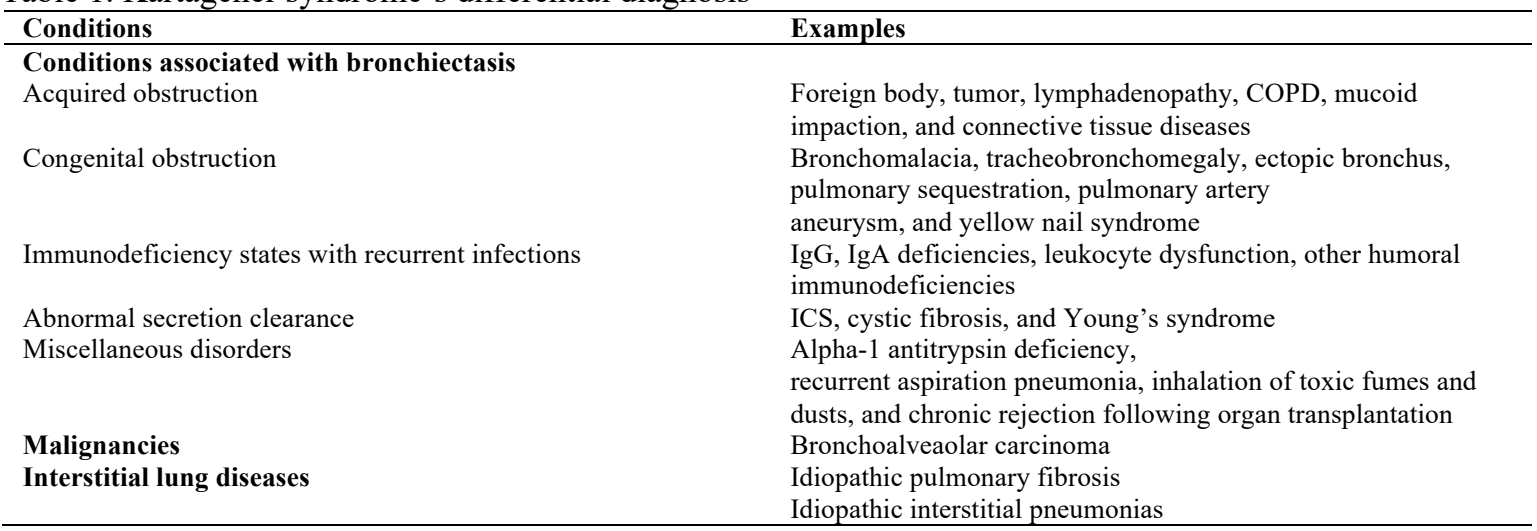

COPD, chronic obstructive pulmonary disease; ICS, immotile-cilia syndrome; IgA, immunoglobulin A; IgG, immunoglobulin G.

\section{Conclusion}

Kartagener syndrome is a subset of PCD characterized by a clinical triad of situs inversus, sinusitis, and bronchiectasis. Adequate understanding of the characteristics of Kartagener syndrome on radiological examinations such as plain x-rays and CT scans will be very helpful in establishing the diagnosis of this case.

\section{References}

Babar, K.S., Khan, H., Ismail, Y., Fawad, M. and Azim, Q., 2013. Kartagener syndrome. Gomal Journal of Medical Sciences, 11(2).

Leigh, M.W., Pittman, J.E., Carson, J.L., Ferkol, T.W., Dell, S.D., Davis, S.D., Knowles, M.R. and Zariwala, M.A., 2009. Clinical and genetic aspects of primary ciliary dyskinesia/Kartagener syndrome. Genetics in Medicine, 11(7), pp.473-487.

Liu, B.C., Huang, T.X. and Liu, C.T., 2020. Kartagener syndrome. The American journal of the medical sciences, 359(6), pp.390-391.

Sarangi, P.K., Acharya, I., Mohanty, J. and Swain, B.M., 2016. Incidentally detected Kartagener's syndrome \{Online\} URL: http://www. eurorad. org/case. php.

Skeik, N. and Jabr, F.I., 2011. Kartagener syndrome. International Journal of General Medicine, 4, p.41. 